\title{
MACROLIDE-LINCOSAMIDE-STREPTOGRAMIN B RESISTANCE AMONG STAPHYLOCOCCUS AUREUS IN CHITWAN MEDICAL COLLEGE TEACHING HOSPITAL, NEPAL
}

\author{
NAVIN KUMAR CHAUDHARY ${ }^{1 *}$, RUSAN PIYA ${ }^{2}$ \\ ${ }^{1}$ Department of Microbiology, Chitwan Medical College, Bharatpur, Nepal. ${ }^{2}$ Department of Laboratory Medicine, Chitwan Medical College, \\ Bharatpur, Nepal. Email: drnavinkumarchaudhary@gmail.com
}

Received: 05 February 2021, Revised and Accepted: 11 March 2021

\section{ABSTRACT}

Objectives: Staphylococcus aureus is often linked with human infection. Clindamycin is one of the key substitute antimicrobial agents in the treatment of $S$. aureus, especially in methicillin-resistant S. aureus (MRSA) infections. Inducible macrolide-lincosamide-streptogramin B (iMLS B) resistance is a crucial factor in antimicrobial susceptibility testing. The intention of the research was to identify S. aureus from distinct clinical specimens and investigate the prevalence of inducible clindamycin resistance among them and also study their association with MRSA.

Methods: A descriptive cross-sectional study was accomplished in the Dept. of Microbiology CMC-TH, Nepal from January 2018 to December 2020 with 525 non-repeated $S$. aureus obtained from a different clinical specimen. Antibiotic susceptibility test was performed by Kirby-Bauer disc diffusion method. MRSA was detected using cefoxitin $(30 \mu \mathrm{g})$ and results were interpreted as stated by CLSI. "D-Test" was done by applying erythromycin $(15 \mu \mathrm{g})$ and clindamycin $(2 \mu \mathrm{g})$ as per CLSI guidelines. Data were analyzed using SPSS IBM version 20.

Results: Among 525 isolates, there were 315 (60.00\%) MRSA. Results of D test analysis showed that 280 (53.33\%) were MLSB sensitive while 245 (46.67\%) were MLSB resistant; where 80 (15.24\%) iMLSB with D zone, 100 (19.05\%) constitutive MLSB (cMLSB) phenotype, and 65 (12.38\%) MS phenotype. Of a total of 80 iMLSB, a significant proportion of $64(80.00 \%)$ was MRSA $(p<0.001)$. All the isolates were sensitive to vancomycin, teicoplanin, and linezolid. The prevalence of both iMLSB and cMLSB was high among MRSA.

Conclusion: In this study, cMLSB phenotype was predominant (19.05\%) followed by iMLSB phenotype (15.25\%) and then MS phenotype (12.38\%). Inducible iMLS B phenotypes, as well as CMLSB, are higher among MRSA. It is advisable to include "D-Test" as a part of regular antibiotic susceptibility testing to detect iMLSB resistance among $S$. aureus.

Keywords: Staphylococcus aureus, Methicillin-resistant Staphylococcus aureus, Clindamycin resistance, Erythromycin resistance, D-Test, Inducible macrolide-lincosamide-streptogramin B, Constitutive MLSB, MS phenotype.

(c) 2021 The Authors. Published by Innovare Academic Sciences Pvt Ltd. This is an open access article under the CC BY license (http://creativecommons.org/ licenses/by/4.0/) DOI: http://dx.doi.org/10.22159/ajpcr.2021v14i5.41012. Journal homepage: https://innovareacademics.in/journals/index.php/ajpcr

\section{INTRODUCTION}

Staphylococci were first identified by Sir Alexander Ogston in 1880 , in pus from a surgical abscess in a knee joint; later in 1884, Freidrich Jukius Rosenbach distinguishes Staphylococcus aureus from Staphylococcus epidermidis [1]. S. aureus is small, ovoid, Grampositive cocci found typically in grape-like clusters or may occur in pairs or singly [2]. It commonly colonizes the skin and noses of healthful individuals. However harmless at these sites, it may get into the body through a crack in the epidermis, for example, erosion, scratch, lesion, surgical procedure, and Foley catheters and give rise to infections [3]. It is accountable for extensive infections including bacterial skin infections, food poisoning, and osteomyelitis; moreover, it is a foremost source of nosocomial infection, extending out of minor skin diseases to a disastrous state such as post-operative wound infection, nosocomial pneumonia, sepsis, and bacterial endocarditis $[4,5]$.

S. aureus infections generally respond to $\beta$-lactam and related classes of antibiotics such as macrolide-lincosamide-streptogramin (MLS) group. Nevertheless, because of the evolution of methicillin resistance out of $S$. aureus isolates, the therapeutics of such infections have become difficult [6]. The rise of methicillin-resistant $S$. aureus (MRSA) among staphylococci is inflation trouble and clindamycin is considered as one of the powerful other agents accessible to address this issue [7]. One of the most key reasons for MRSA evolution is redundant and wide ranging antibiotic overuse for less severe infections. Unluckily these MRSA isolates which are susceptible only to glycopeptides antibiotics such as vancomycin are becoming multidrug resistant (MDR) [8]. The rise in the number of MDR strains has led to a renewed curiosity in the utilization of macrolide (e.g., erythromycin)-lincosamide (e.g., clindamycin)-streptogramin B (e.g., quinupristin/dalfopristin) (collectively called as MLSB family) antibiotics to treat $S$. aureus infections with clindamycin being the favorable agent because of its superb pharmacokinetic properties [9].

Staphylococcal strains resistant to MLSB antibiotics have escalated in number after the extensive use of these antibiotics for treating serious staphylococcal infections [10]. The most usual process for such resistance is target site modification mediated by erm genes, which can be demonstrated either constitutively (cMLSB phenotype) or inducible (iMLSB phenotype). The erm genes code for methylase enzyme which methylates and alters the target site of MLSB antibiotics, that is, the 23S ribosomal RNA [11]. Active efflux pump encoded by msr A gene (MS phenotype) is another process of resistance [12]. $S$. aureus with constitutive resistance show resistance to erythromycin and clindamycin on in vitro testing, whereas isolates with inducible resistance show resistance to erythromycin but seem susceptible to clindamycin on disc diffusion test. Inducible clindamycin resistance in staphylococci can be detected by D test [13]. It is advocated that an accurate proportion of clindamycin resistance is being misjudged, especially for laboratories where testing for inducible resistance is not routinely done. For this reason, it is recommended that microbiology laboratories must accomplish the D-zone test on all staphylococcal strains that are erythromycin resistant and clindamycin sensitive, 
before communicating clindamycin sensitive, to ascertain those strains that perhaps resistant in the course of treatment [14]. Failure to point out iMLSB resistance may accelerate the clinical failure of clindamycin treatment [15].

The incidence of clindamycin resistance varies from place to place and therefore a local data are important to guide empirical treatment [16]. In Nepal, few reports on the prevalence of inducible clindamycin resistance among $S$. aureus have been published [17,18]. Data describing the prevalence of clindamycin resistance among clinical isolates of $S$. aureus and MRSA are lacking in our geographic area. Thus, the present study was accomplished to determine the prevalence of inducible clindamycin resistance among $S$. aureus isolates and also to study their association with MRSA in our set up.

\section{METHODS}

The study was conducted in the Department of Microbiology CMCTH, Nepal, from January 2018 to December 2020 with 525 nonrepetitive $S$. aureus isolated from distinct clinical specimens. Antibiotic susceptibility test was performed by Kirby-Bauer disc diffusion method $[19,20]$. Based on the hospital antibiotic policy, the following antibiotics were used, namely, amikacin $(30 \mu \mathrm{g})$, amoxiclav $(30 \mu \mathrm{g})$ (amoxicillin/clavulanic acid 20/10 $\mu \mathrm{g}$ ), cefoxitin (30 $\mu \mathrm{g}$ ), ceftriaxone $(30 \mu \mathrm{g})$, ciprofloxacin $(5 \mu \mathrm{g})$, clindamycin $(2 \mu \mathrm{g})$, cotrimoxazole $(25 \mu \mathrm{g})$ (trimethoprim/sulfamethoxazole 1.25/3.75 $\mu \mathrm{g}$ ), erythromycin (15 $\mu \mathrm{g}$ ), gentamicin $(10 \mu \mathrm{g})$, cloxacillin $(5 \mu \mathrm{g})$, linezolid $(30 \mu \mathrm{g})$, penicillin (10 units), piperacillin/tazobactam $(100 / 10 \mu \mathrm{g})$, tigecycline $(15 \mu \mathrm{g})$, tetracycline $(30 \mu \mathrm{g})$, teicoplanin $(30 \mu \mathrm{g})$, and vancomycin $(30 \mu \mathrm{g})$. Sensitivity testing of vancomycin and teicoplanin was done by the minimum inhibitory concentration method. All antimicrobial drugs were obtained from HiMedia Laboratories, India. Screening of MRSA was done using cefoxitin $(30 \mu \mathrm{g})$. If the cefoxitin $(30 \mu \mathrm{g})$ zone of inhibition was less than $21 \mathrm{~mm}$, it was reported as MRSA (Fig. 1) [19].

Inducible clindamycin resistance was detected using erythromycin (15 $\mu \mathrm{g})$ and clindamycin $(2 \mu \mathrm{g})$ as per CLSI guideline [20]. Three different types of the phenotype were appreciated and interpreted. Inducible MLSB (iMLSB)

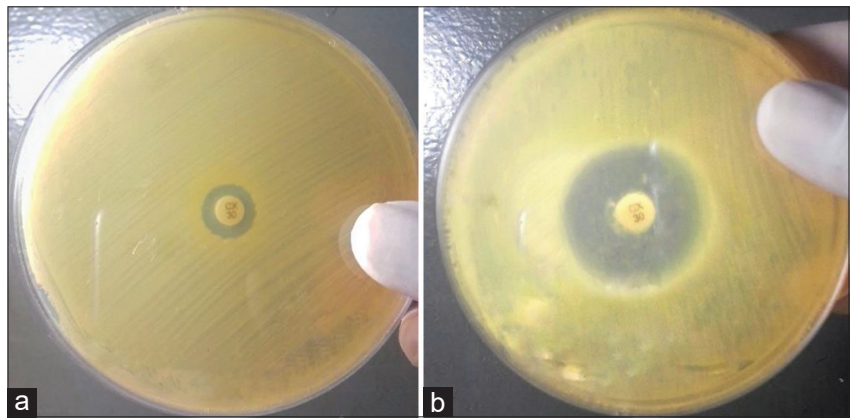

Fig. 1: Detection of methicillin-resistant Staphylococcus aureus using cefoxitin disk. (a) Methicillin-resistant $S$, aureus (b) Methicillin-sensitive $S$, aureus phenotype: Staphylococcal isolates showing resistance to erythromycin while being sensitive to clindamycin and giving a $\mathrm{D}$-shaped zone of inhibition around clindamycin with flattening toward erythromycin disc. Constitutive MLSB (cMLSB) phenotype: Those staphylococcal isolates, which showed resistance to both erythromycin and clindamycin with the circular shape of the zone of inhibition, if any around clindamycin. MS phenotype: Isolates exhibiting resistance to erythromycin and sensitivity to clindamycin and giving a circular zone of inhibition around clindamycin (Fig. 2).

To verify that the susceptibility result is accurate, control strain of S. aureus American type culture collection (ATCC) 25293 was streaked on the prepared media plates and observed for significant growth. Control strains of E. coli (ATCC 25922), S. aureus (ATCC 25923), and $P$. aeruginosa (ATCC 27853) were used for the standardization of the Kirby-Bauer test and also for the correct interpretation of the zone of inhibition. For MRSA test standardization, S. aureus strains ATCC 25923 were used as negative and ATCC 43300 were used as positive controls. Qualities of each agar plate were tested by incubating one plate of each batch on the incubator overnight without inoculating. The collected data were summarized, presented, and analyzed using the software SPSS version 20 (Chicago, USA). Qualitative data were summarized as frequency and percentages. $\mathrm{p}<0.05$ was considered statistically significant. Ethical approval was taken from Chitwan Medical College (CMC) - Institutional Review Committee. Informed consent was taken from the patient before their inclusion in the research.

\section{RESULTS}

Demographic distribution and identification of bacterial isolates A total of 525 non-repetitive $S$. aureus were collected from the different specimens. Among them, 420 (80.00\%) were from pus, $42(8.00 \%)$ from blood, 26 (4.95\%) from sputum, 21 (4.00\%) from urine, and 16 (3.05\%) from body fluid and tissue, respectively. Isolation frequency of $S$. aureus was $273(52.00 \%)$ and $252(48.00 \%)$ among males and females, respectively. S. aureus was dominant in IPD patient $315(60.00 \%)$ than OPD patient $210(40.00 \%)$. Among IPD patient, the growth of $S$. aureus was predominant in surgical ward 157 (29.90\%) followed by medicine ward 82 (15.62\%), orthopedic ward 65 (12.38\%), medical/pediatric/ neonatal ICU and CCU 63 (12.00\%), pediatric ward 53 (10.10\%), ENT ward $42(8.00 \%)$, gynecology ward $42(8.00 \%)$, and tropical ward 21 (4.00\%). Out of 525 S. aureus, 315 (60.00\%) were MRSA. MRSA isolates were isolated more from pus 257 (48.95\%) as compared to other samples. The distribution of MRSA was found to be dominant in IPD patients 195 (37.14\%) compared to 120 (22.86\%) among OPD patients. Results of D test analysis showed that out of 525 S. aureus, 80 (15.24\%) showed a D zone; where $280(53.33 \%)$ were MLSB sensitive while 245 (46.67\%) were MLSB resistant. In this study, cMLSB phenotype was predominant $100(19.05 \%)$ followed by the iMLSB phenotype 80 (15.25\%) and then MS phenotype 65 (12.38\%), as shown in Fig. 3.

iMLSB phenotype as well as cMLSB was higher among MRSA 64 $(20.32 \%)$ and $79(25.08 \%)$ as compared to MSSA $16(7.62 \%)$ and 21 $(10.00 \%)$, respectively (Chi-square test, $\mathrm{p}<0.001$ ), as expressed in Table 1.
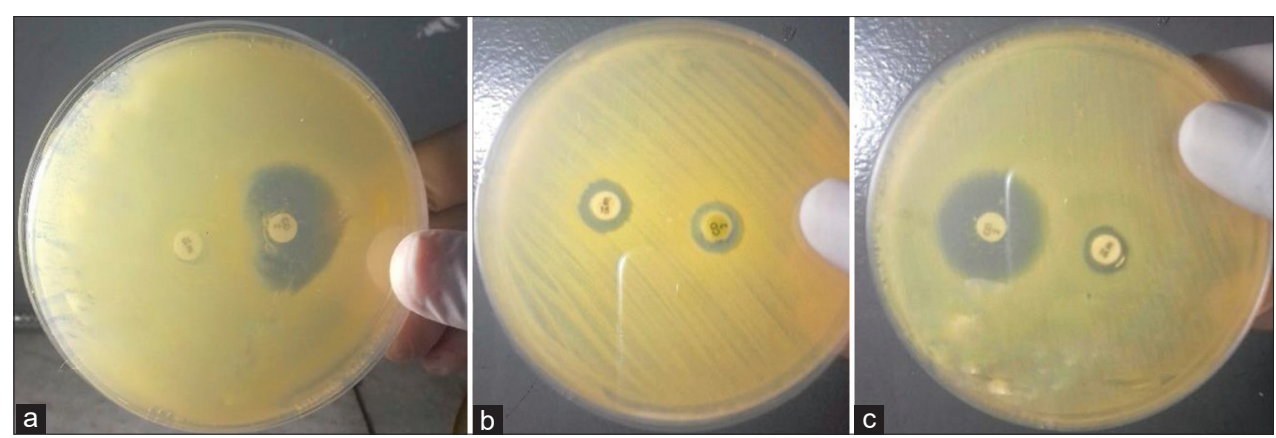

Fig. 2: Different MLSB phenotype. (a) iMLSB phenotype. (b) cMLSB phenotype. (c) MS phenotype 
Table 1: Susceptibility pattern against erythromycin and clindamycin among MRSA and MSSA isolates ( $\mathrm{n}=525)$

\begin{tabular}{lllllll}
\hline Isolates & iMLSB (\%) & cMLSB (\%) & MS (\%) & MLSB sensitive (\%) & Total (\%) & p-value \\
\hline MRSA & $64(12.19)$ & $79(14.80)$ & $52(9.90)$ & $120(22.86)$ & $315(60.00)$ & p<0.001 \\
MSSA & $16(3.05)$ & $21(4.00)$ & $13(2.48)$ & $160(30.48)$ & $210(40.00)$ & $525(100.00)$ \\
Total & $80(15.24)$ & $100(19.05)$ & $65(12.38)$ & $280(53.33)$ & \\
\hline
\end{tabular}

\section{Antibiotic sensitivity profile}

$\beta$-lactams, sulfonamides, quinolones, aminoglycosides, tetracycline, glycopeptides, macrolide, lincosamide, and oxazolidinones groups of antibiotics were tested against $S$. aureus isolates. All the isolates $(100 \%)$ were sensitive to vancomycin, teicoplanin, and linezolid. Among antibiotics of $\beta$-lactam class, penicillin was least effective with only $14.29 \%$ sensitivity. Cloxacillin showed $42.86 \%$, cefoxitin showed $41.41 \%$, and amoxicillin/clavulanic acid showed $40.00 \%$ sensitivity. Other antibiotics of the same class showed a sensitivity rate of less than $40 \%$. Another antibiotic with a higher sensitivity rate was amikacin $88.00 \%$. Gentamicin, being from the same class of antibiotics (aminoglycosides), had $48.95 \%$ sensitivity. Ciprofloxacin was the least effective with a sensitivity rate of $31.05 \%$. Cotrimoxazole was $58.86 \%$ sensitive. Erythromycin and clindamycin both had more than 50\% sensitivity rate, that is, $52.95 \%$ and $66.10 \%$, respectively. Antibiotic from the group tetracycline was also effective. Tetracycline had 55.05\% sensitivity while tigecycline had $44.95 \%$ sensitivity. Detail summary of the susceptibility pattern of $S$. aureus isolates is shown in Table 2.

\section{DISCUSSION}

Screening for antimicrobial susceptibility among the clinical bacterial isolates is important for the best result of the treatment. In our study, of 525 isolates of $S$. aureus, $80.00 \%$ were from the pus sample, which signifies their leading role in abscess formation. Antimicrobial resistance has been observed as one of the supreme microbial threats of this century [21]. One of the crucial issues while treating infection caused by $S$. aureus is due to the multidrug resistance plus the emergence of methicillin resistance [22]. Clindamycin is commonly used for the treatment of skin and soft-tissue infections caused by $S$. aureus. However, S. aureus with inducible clindamycin resistance is increasing day by day, form such mutant's constitutive resistance can arise spontaneously during clindamycin therapy [23]. Therefore, all the clinical isolates of $S$. aureus must be checked for inducible resistance (D-test) before clindamycin is reported as susceptible and to prevent therapeutic failure [24]. In the present study, the overall prevalence of inducible clindamycin resistance (iMLSB) among the clinical isolates of $S$. aureus was $15.24 \%$ which is near to the report of Singh et al., $13.39 \%$ [25], Ansari et al., 12.4\% [26], and Sah et al., 12.1\% [17] from Nepal, Parasa et al., 15.03\% from India [27], and Van der Heijden et al., $12.3 \%$ from Brazil [28]. Different prevalence rates of iMLSB have been reported in other studies; Mohapatra et al., 18.2\% [29], Raut et al., 25.6\% [30], Shrestha et al., 20.6\% [31] from Nepal, and Lall et al., 20.30\% [32] from India. Higher iMLSB prevalence of 45\% from Germany [33] and 62\% from the US [34] has also been reported. Constitutive clindamycin resistance (cMLSB) phenotype was found to be $19.50 \%$ in the present study, which is low as compared to another study $[23,29,31,32]$. Such disparity could be because of variation in a study period, group of patients, and geographical site. The present study demonstrated a higher prevalence of both iMLSB and cMLSB among MRSA as compared to MSSA. This finding is consistent with other reports [17,25,29,31,32]. However, Schreckenberge et al. [16], Levin et al. [35], and Marr et al. [36] reported a higher incidence of iMLSB among MSSA. In the present study, MRSA was dominant in male (31.2\%) while MSSA was dominant in female (21.1\%); a similar study was conducted by Raut et al. reported $52.3 \%$ and $64.3 \%$, respectively [30]. In our study, MRSA was found to be dominant (47.7\%) in the pus sample which is consistent with the reports of other authors, Raut et al. (49.2\%) [30], Pandey et al. (28.73\%) [37], and Shrestha et al. (82.6\%) [31]. However, in the study by Thapa et al. [38], S. aureus was dominant in blood sample $44.60 \%$ while $23.40 \%$ was in pus.
Table 2: Antibiotic susceptibility pattern of Staphylococcus aureus $(\mathrm{n}=525)$

\begin{tabular}{|c|c|c|c|c|c|}
\hline \multirow{3}{*}{$\begin{array}{l}\text { Class of } \\
\text { antibiotics }\end{array}$} & \multirow{3}{*}{$\begin{array}{l}\text { Antibiotics } \\
\text { used }\end{array}$} & \multicolumn{4}{|c|}{ Susceptibility pattern } \\
\hline & & \multicolumn{2}{|c|}{ Resistant } & \multicolumn{2}{|c|}{ Sensitivity } \\
\hline & & No. & $\%$ & No. & $\%$ \\
\hline \multirow{2}{*}{ Aminoglycosides } & Amikacin & 63 & 12.00 & 462 & 88.00 \\
\hline & Gentamicin & 268 & 51.56 & 257 & 48.95 \\
\hline \multirow[t]{6}{*}{$\beta$-lactams } & $\begin{array}{l}\text { Amoxicillin/ } \\
\text { clavulanic acid }\end{array}$ & 315 & 60.00 & 210 & 40.00 \\
\hline & Penicillin & 450 & 85.71 & 75 & 14.29 \\
\hline & Cefoxitin & 309 & 58.86 & 216 & 41.14 \\
\hline & Ceftriaxone & 325 & 61.90 & 200 & 38.10 \\
\hline & Cloxacillin & 300 & 57.14 & 225 & 42.86 \\
\hline & $\begin{array}{l}\text { Piperacillin/ } \\
\text { tazobactam }\end{array}$ & 320 & 60.95 & 205 & 39.05 \\
\hline \multirow[t]{2}{*}{ Glycopeptides } & Vancomycin & 00 & 00.00 & 525 & 100.00 \\
\hline & Teicoplanin & 00 & 00.00 & 525 & 100.00 \\
\hline Lincosamides & Clindamycin & 178 & 33.90 & 347 & 66.10 \\
\hline Macrolides & Erythromycin & 247 & 47.05 & 278 & 52.95 \\
\hline Oxazolidinones & Linezolid & 00 & 00.00 & 525 & 100.00 \\
\hline Quinolones & Ciprofloxacin & 362 & 68.95 & 163 & 31.05 \\
\hline Sulfonamides & Cotrimoxazole & 216 & 41.14 & 309 & 58.86 \\
\hline \multirow[t]{2}{*}{ Tetracyclines } & Tigecycline & 289 & 55.05 & 236 & 44.95 \\
\hline & Tetracycline & 236 & 44.49 & 289 & 55.05 \\
\hline
\end{tabular}

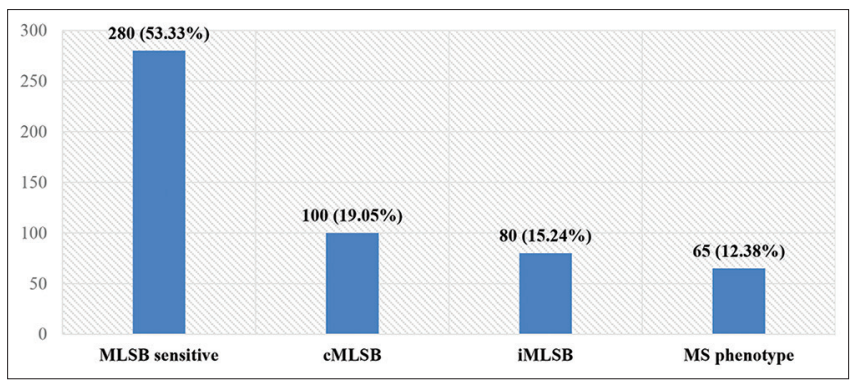

Fig. 3: Susceptibility pattern against erythromycin and clindamycin among Staphylococcus aureus isolates $(n=525)$

This study demonstrated that overall rates of susceptibility pattern of $S$. aureus to the commonly used antibiotics were less than $65 \%$, except clindamycin, amikacin, vancomycin, linezolid, and teicoplanin. A high proportion of isolates, $85.93 \%$ were resistant to penicillin. This result was expected as only a few strains of $S$. aureus do not produce beta-lactamases [26]. Ciprofloxacin being cheap and easily available antibiotics it has been widely used to treat against $S$. aureus. In this study, resistance rate of ciprofloxacin was $69.53 \%$, which is comparatively higher than a report of Ansari et al., 63.7\% [26]. Some other researchers found a resistance rate of ciprofloxacin to be $32.73 \%$ by Sanjana et al. [39] and $11 \%$ by Baral et al. [40]. The rate of resistance of cotrimoxazole in our study was $41.41 \%$ which is similar to a study by Shrestha et al. [31]. Baral et al. found $64 \%$ rate of resistance to cotrimoxazole which is higher than in our study [40]. Similarly, gentamicin was $51.56 \%$ resistant in our study which is comparable to $54.50 \%$ found by Mishra et al. [41]. Fortunately, the rate of resistance to amikacin is low at $11.71 \%$. All the isolates $(100 \%)$ in the present study were susceptible to vancomycin and teicoplanin, this report is consistent with other studies [25,26,39-41], this proves that glycopeptides should be used as empiric therapy for serious staphylococcal infections. 
Absolutely susceptibility of this drug in Nepal might be related to the low use of this agent due to its high cost [26]. Linezolid is another drug to have $100 \%$ susceptibility which is consistent with the report of Singh et al. [25] and Raut et al. [30].

\section{CONCLUSION}

In this study, cMLSB phenotype was predominant (19.05\%) followed by iMLSB phenotype $(15.25 \%)$ and then MS phenotype (12.38\%). Inducible MLS B phenotypes, as well as CMLSB, are higher among MRSA. Clindamycin, which has outstanding bone and tissue penetration along with its ability to accumulate in an abscess, has become one of the beneficial antibiotics to treat $S$. aureus infection. Therefore, D-test should be performed routinely and the clinician should be enlightened regarding the likely failure of clindamycin therapy in infections caused by $S$. aureus harboring iMLSB resistance.

\section{ACKNOWLEDGMENTS}

We would like to thank the technician of the Department of Microbiology, $\mathrm{CMC}-\mathrm{TH}$, for their assistance and the entire patient for participation in this study.

\section{AUTHORS' CONTRIBUTIONS}

NKC: Concept and design of the study, reporting of test results, collection, analysis and interpretation of data, and drafting the final version of the manuscript. RP: Collection, processing of specimens, and preparation of manuscript.

\section{CONFLICTS OF INTEREST}

There are no conflicts of interest regarding the publication of this article.

\section{AUTHORS' FUNDING}

The authors did not receive any funding for this research work.

\section{REFERENCES}

1. Licitra G. Etymologia Staphylococcus. Emerg Infect Dis 2013;19:1553.

2. Taylor TA, Unakal CG. Staphylococcus aureus. Treasure Island, FL: StatPearls Publishing; 2021.

3. Patel P, Khandelwal N, Raval P, Patel B, Soni S, Vegad M. Prevalence and antimicrobial susceptibility pattern of methiciliin resistant Staphylococcus aureus in tertiary care teaching hospital-Western India. Int J Med Sci Public Health 2014;3:58-60.

4. Forbes BA, Sahm DF, Weissfeld AS, Bailey WR. Bailey and Scott's Diagnostic Microbiology. $12^{\text {th }}$ ed. St Louis: Elsevier Mosby; 2007. p. 254-64.

5. Chambers HF. The changing epidemiology of Staphylococcus aureus? Emerg Infect Dis 2001;7:178-82.

6. Kluytmans J, Belkum AV, Verbrugh H. Nasal carriage of Staphylococcus aureus: Epidemiology, underlying mechanisms, and associated risks. Clin Microbiol Rev 1997;10:505-20.

7. Fokas S, Fokas S, Tsironi M, Kalkani M, Dionysopouloy M. Prevalence of inducible clindamycin resistance in macrolide-resistant Staphylococcus spp. Clin Microbiol Infect 2005;11:337-40.

8. Sasirekha B. Prevalence of ESBL, AmpC $\beta$-lactamases and MRSA among uropathogens and its antibiogram. EXCLI J 2013;12:81-8.

9. Mama M, Aklilu A, Misgna K, Tadesse M, Alemayehu E. Methicillin and inducible clindamycin resistant Staphylococcus aureus among patients with wound infection attending Arba Minch hospital, South Ethiopia. Hindawi Int J Micro 2019;2965490:1-9.

10. Delialioglu N, Aslan G, Ozturk C, Baki V, Sen S, Emekdas G. Inducible clindamycin resistance in staphylococci isolated from clinical samples. Jpn J Infect Dis 2005;58:104-6.

11. Blair JM, Webber MA, Baylay AJ, Ogbolu DO, Piddock LJ. Molecular mechanisms of antibiotic resistance. Nat Rev Microbiol 2015;13:42-51.

12. Jindal N, Singh S, Grover P, Malhotra R. Prevalence of inducible clindamycin resistance among clinical isolates of MRSA in Malwa region of Punjab (North India). Indian J Res 2013;2:133-4.

13. Wayne P. Clinical and Laboratory Standards Institute: Performance Standards for Antimicrobial Susceptibility Testing. 20 $0^{\text {th }}$ Informational
Supplement. CLSI Document M100-S20. Wayne, PA: Clinical and Laboratory Standards Institute; 2010.

14. Maltezou HC, Giamarellou H. Community-acquired methicillinresistant Staphylococcus aureus infections. Int J Antimicrob Agents 2006;27:87-96.

15. Drinkovic D, Fuller ER, Shore KP, Holland DJ, Ellis-Pegler R. Clindamycin treatment of Staphylococcus aureus expressing inducible clindamycin resistance. J Antimicrob Chemother 2001;48:315-6.

16. Schreckenberger PC, Ilendo E, Ristow KL. Incidence of constitutive and inducible clindamycin resistance in Staphylococcus aureus and coagulase-negative staphylococci in a community and a tertiary care hospital. J Clin Microbiol 2004;42:2777-9.

17. Sah P, Khanal R, Lamichhane P, Upadhaya S, Lamsal A, Pahwa V. Inducible and constitutive clindamycin resistance in Staphylococcus aureus: An experience from Western Nepal. Int J Biomed Res 2015;6:316-9

18. Amatya R, Shrestha R. Incidence of macrolide lincosamide streptogramin B resistance in coagulase negative staphylococci from a tertiary care hospital in Nepal. Nepal Med Coll J 2015;17:157-60.

19. Wayne P. Clinical and Laboratory Standards Institute: Methods for Dilution Antimicrobial Susceptibility Test for Bacteria that Grow Aerobically; Approved Standard. 10 $0^{\text {th }}$ ed. CLSI Document M07-A10. Wayne, PA: Clinical and Laboratory Standards Institute; 2015.

20. Wayne P. Clinical and Laboratory Standards Institute: Performance Standards for Antimicrobial Disk Susceptibility Tests; Approved Standard. $12^{\text {th }}$ ed. CLSI Document M02-A12. Wayne, PA: Clinical and Laboratory Standards Institute; 2015.

21. Smolinski MS, Hamburg MA, Lederberg J. Microbial Threats to Health: Emergence, Detection and Response. Washington, DC: National Academies Press; 2003.

22. Waldvogel FA. Mandell, Douglas, Bennett's Principle and Practice of Infectious Diseases. $5^{\text {th }}$ ed. Philadelphia, PA: Churchill Livingstone; 2000.

23. Yilmaz G, Aydin K, Iskender S, Caylan R, Koksal I. Detection and prevalence of inducible clindamycin resistance in staphylococci. J Med Microbiol 2007;56:342-5.

24. Perez LR, Caierao J, Antunes AL, d'Azevedo PA. Use of the D test method to detect inducible clindamycin resistance in coagulase negative staphylococci (CoNS). Braz J Infect Dis 2007;11:186-8.

25. Singh GK, Chaudhari BK, Parajuli KP. Phenotypic study of macrolidelincosamide-streptogramin B resistance in Staphylococcus aureus and their relationship with methicillin-resistant Staphylococcus aureus (MRSA) at tertiary care in Eastern Nepal. J Nob Med Coll 2016;5:1-5.

26. Ansari S, Nepal HP, Gautam R, Rayamajhi N, Shrestha S, Upadhyay G, et al. Threat of drug resistant Staphylococcus aureus to health in Nepal. BMC Infect Dis 2014;14:157.

27. Parasa LS, Tumati SR, Chigurupati SP, Parabathina RK, Santhisree K, Kumar LC, et al. Prevalence of induced clindamycin resistance in methicillin resistant Staphylococcus aureus from hospital population of coastal Andhara Pradesh, South India. Arch Clin Microbiol 2011;2:1-6.

28. Van der Heijden I, Sinto S, Oplustil S, Mendes C, editors. Occurrence of MLS Resistance in Staphylococcal and Streptococcol Clinical Isolates. Washington DC: Abstract A-86. $101^{\text {st }}$ General Meeting of the American Society for Microbiology; 2001.

29. Mohapatra T, Shrestha B, Pokhrel BM. Constitutive and inducible clindamycin resistance in Staphylococcus aureus and their association with meticillin-resistant S. aureus (MRSA): Experience from a tertiary care hospital in Nepal. Int J Antimicrob Agents 2009;33:187-9.

30. Raut S, Bajracharya K, Adhikari J, Pant SS, Adhikari B. Prevalence of methicillin resistant Staphylococcus aureus in Lumbini Medical College and Teaching Hospital, Palpa, Western Nepal. BMC Res Notes 2017; 10:187.

31. Shrestha B, Pokhrel BM, Mohapatra TM. Phenotypic characterization of nosocomial isolates of Staphylococcus aureus with reference to MRSA. J Infect Dev Ctries 2009;3:554-60.

32. Lall M, Sahni A. Prevalence of inducible clindamycin resistance in Staphylococcus aureus isolated from clinical samples. Med J Armed forces India 2014;70:43-7.

33. Schmitz FJ, Petridou J, Fluit A, Hadding U, Peters G, Von Eiff C, et al. Distribution of macrolide-resistance genes in Staphylococcus aureus blood-culture isolates from fifteen German university hospitals. Eur J Clin Microbiol Infect Dis 2000;19:385-7.

34. Sanchez ML, Flint KK, Jones RN. Occurrence of macrolidelincosamide-streptogramin resistances among staphylococcal clinical isolates at a university medical center: Is false susceptibility to new macrolides and clindamycin a contemporary clinical and in vitro testing problem? Diagn Microbiol Infect Dis 1993;16:205-13. 
35. Levin TP, Suh B, Axelrod P, Truant AL, Fekete T. Potential clindamycin resistance in clindamycin-susceptible, erythromycin-resistant Staphylococcus aureus: Report of a clinical failure. Antimicrob Agents Chemother 2005;49:1222-4

36. Marr JK, Lim AT, Yamamoto L. Erythromycin-induced resistance to clindamycin in Staphylococcus aureus. Hawaii Med J 2005; 64:6-8.

37. Pandey S, Raza M, Bhatta C. Prevalence and antibiotic sensitivity pattern of methicillin-resistant-Staphylococcus aureus in Kathmandu medical college teaching hospital. J Inst Med 2013;34:13-7.

38. Thapa S, Sapkota LB. Prevalence of inducible clindamycin resistance in erythromycin resistant clinical isolates of Staphylococcus aureus and
CONS at tertiary care hospital. JCMS Nepal 2016;12:83-8

39. Sanjana R, Shah R, Chaudhary N, Singh Y. Prevalence and antimicrobial susceptibility pattern of methicillin-resistant Staphylococcus aureus (MRSA) in CMS-teaching hospital: A preliminary report. JCMS Nepal 2010;6:1-6.

40. Baral R, Khanal B, Acharya A. Antimicrobial susceptibility patterns of clinical isolates of Staphylococcus aureus in Eastern Nepal. Health Renaissance 2011;9:78-82.

41. Mishra SK, Rijal BP, Pokhrel BM. Emerging threat of multidrug resistant bugs-Acinetobacter calcoaceticus baumannii complex and methicillin resistant Staphylococcus aureus. BMC Res Notes 2013;6:98. 